ADVANCES IN

PSYCHIATRY \& NEUROLOGY

POSTĘPY

Psychiatrii i Neurologi

Correspondence to:

Dr. Nattakarn Limphaibool

Poznan University of Medical Sciences

Poznan, Poland

e-mail: tongn.lim@gmail.com

Submitted: 09.03.2021

Accepted: 07.05.2021

\section{Musical hallucinations in cerebrovascular disease}

\author{
Nattakarn Limphaibool (D), Barbara Maciejewska, \\ Piotr Kowal, Wojciech Kozubski, Piotr Iwanowski
}

Poznan University of Medical Sciences, Poznan, Poland

\begin{abstract}
Purpose: Musical hallucinations $(\mathrm{MH})$ are a subset of complex auditory hallucinations in which individuals perceive music in the absence of an external auditory stimulus. It is a rare phenomenon, first described by Ballinger in 1846, with diverse presentations from familiar childhood melodies to a simple pitch which evolved into the harmonies Robert Schumann incorporated in his sole Violin Concerto.

Views: This uncommon phenomenon has diverse etiologies, including psychiatric and neurological backgrounds, which guide its classification and methods of treatment. The pathophysiological basis of MH remains understood incompletely, potentially resulting from lesions anywhere along the auditory pathway, from the external auditory canal to the auditory cortex. The strong association between $\mathrm{MH}$ and hearing impairment has led researchers to hypothesize that $\mathrm{MH}$ represent a "release phenomenon," in which sensory deprivation, eliminating the afferent input to the auditory sensory network, instigates spontaneous activity within a system - comparable to the Charles Bonnet syndrome, in which visual impairment precipitates the development of visual hallucinations (so called auditory Charles Bonnet syndrome), and phantom limb syndrome, in which amputees experience sensations in a limb that is not no longer there. In this paper, we report on six cases of $\mathrm{MH}$ in patients with cerebrovascular disease, who presented to the neurology department at the Poznan University of Medical Sciences from 2015 to 2018.

Conclusions: We discuss the findings of computed tomography and magnetic resonance imaging of six cases of $\mathrm{MH}$ in patients with cerebrovascular disease, and the treatment leading to its resolution. We briefly review the literature on $\mathrm{MH}$ in patients with cerebrovascular diseases, discussing their suggested pathophysiology, clinical presentations and response to medical treatment.
\end{abstract}

Key words: stroke, cerebrovascular disease, hearing impairment, brain lesion, musical hallucinations.

Musical hallucinations $(\mathrm{MH})$ are a subset of complex auditory hallucinations in which individuals perceive music in the absence of an external auditory stimulus [1]. Diverse presentations of $\mathrm{MH}$ have been reported, from familiar childhood melodies to a simple pitch which evolved into the harmonies Robert Schumann incorporated in his sole Violin Concerto [2]. This uncommon phenomenon has diverse psychiatric and neurological etiologies which guide its classification and methods of treatment. The pathophysiological basis of $\mathrm{MH}$ remains understood incompletely, potentially resulting from lesions anywhere along the auditory pathway, from the external auditory canal to the auditory cortex. The strong association between $\mathrm{MH}$ and hearing impairment has led researchers to hypothesize that $\mathrm{MH}$ represent a "release phenomenon", in which sensory deprivation eliminating the afferent input to the auditory sensory network instigate spontaneous activity within a system which is comparable to the Charles Bonnet syndrome where visual impairment precipitates the development of visual hallucinations (so-called auditory Charles Bonnet syndrome), and phantom limb syndrome, in which amputees experience sensations in a limb that is no longer there $[1,3]$.

We report on six cases of $\mathrm{MH}$ in patients with cerebrovascular disease, who presented to the neurology department at Poznan University of Medical Sciences from 2015 to 2018. Their computed tomography (CT) and magnetic resonance imaging (MRI) are discussed along with the treatment leading to the resolution of $\mathrm{MH}$. We also briefly review the literature of $\mathrm{MH}$ in patients with cerebrovascular diseases, discussing the proposed pathophysiology, clinical presentations and response to medical treatment. Patients' informed consent was waived due to the observational and retrospective design of this study. 
Patient 1: A 78-year-old female patient with a history of arterial hypertension and a past myocardial infarction was admitted to the Department of Phoniatrics and Audiology due to a sudden deterioration in her hearing over the previous 3 days. The patient reported auditory hallucinations, which she described as fragments of different songs, most often from the Polish version of 'Happy birthday. Her physical examination revealed vertical nystagmus, dysdiadochokinesia, hearing loss, and a positive Romberg sign. Audiological tests showed profound bilateral sensorineural hearing loss with an auditory threshold at the $90-$ to $100-\mathrm{dB}$ range. CT imaging revealed diffuse ischemic brain changes. Brain MRI showed chronic, diffuse ischemic changes in the white matter of both hemispheres (primarily in the frontal and parietal lobes) with a maximal diameter of $5 \mathrm{~mm}$ in FLAIR and T2-weighted sequences (Figure I). Magnetic resonance angiography (MRA) showed atherosclerotic lesions within the internal carotid arteries and its branches. The administration of methylprednisolone $1 \mathrm{~g}$ /day resulted in an improvement in hearing and a significant reduction of $\mathrm{MH}$ after 5 days. The patient was given hearing aids for both ears. No auditory hallucinations were reported by the patient throughout the audiological and neurological examinations in the 3-year follow-up.

Patient 2: A 63-year-old female patient with a history of arterial hypertension presented with a sudden, transient episode of aphasia. A diagnosis of transient ischemic attack (TIA) was made. As the patient regained her ability to speak, she reported the appearance of $\mathrm{MH}$ in the form of a constant replaying of popular folk songs.

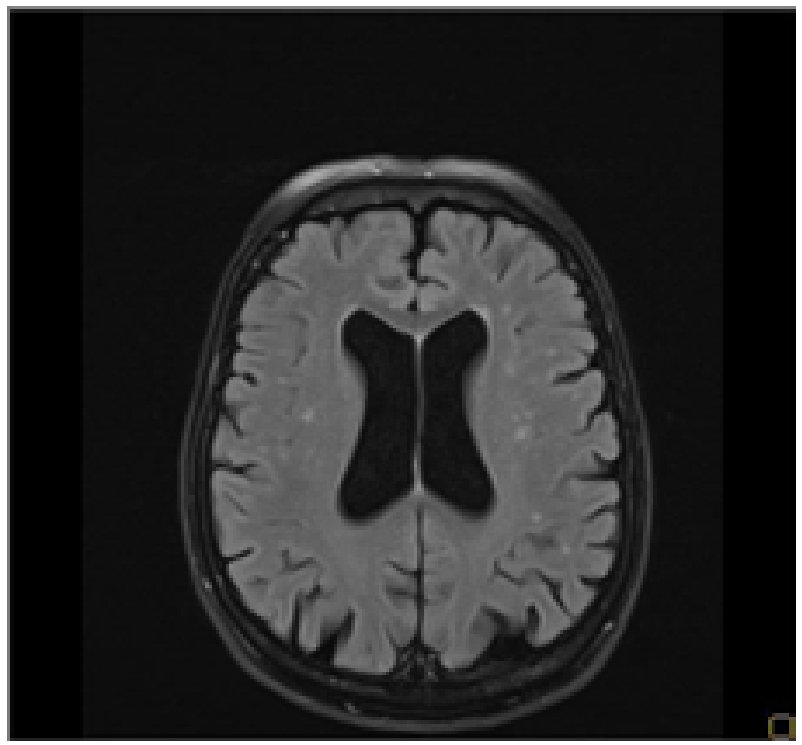

Figure I. Magnetic resonance imaging of the brain of a 78-year-old patient showing diffuse ischemic changes, disseminated in time, in the frontal and parietal lobes in FLAIR
The patient displayed moderate bilateral sensorineural hearing loss on physical examination. Neurological examination was otherwise unremarkable. CT imaging showed disseminated ischemic changes, and MRI revealed disseminated ischemic cortical and subcortical lesions in both hemispheres. There were no DWI-positive lesions. In the 1-month follow-up, the patient reported a shift from folk songs to fragments of orchestral music and choral singing. To reduce the hallucinations carbamazepine $400 \mathrm{mg} /$ day was administered which alleviated her symptoms. In the following months, however, sounds of dogs' barking replaced the prior $\mathrm{MH}$. The carbamazepine dose was increased to $800 \mathrm{mg} /$ day. The patient reported prolonged periods of silence in the following6 months. 9 months later, the patient reported only tinnitus without the presence of auditory hallucinations.

Patient 3: A 75-year-old female patient with underlying arterial hypertension was urgently admitted to the Department of Neurology due to dysarthria, left-sided facial drooping, and left upper limb weakness. She reported $\mathrm{MH}$ which she recalled as being the melodies she had heard as a young girl. Pleasant memories resurfaced in connection with her MH. Head MRI revealed diffuse ischemic changes which were disseminated in time, and an acute ischemic lesion in the right temporal lobe. Auditory testing showed moderate sensorineural hearing loss with an auditory threshold at the 50 - to $60-\mathrm{dB}$ range. Due to the hallucinations, carbamazepine $400 \mathrm{mg}$ /day and clonazepam $1 \mathrm{mg} /$ day were administered. This resulted in a fluctuation of symptoms over the following 6 weeks, and finally in the resolution of $\mathrm{MH}$.

Patient 4: A 52-year-old male patient with a history of arterial hypertension presented to the emergency unit with sudden-onset severe headache which began in the past few hours. CT revealed bleeding in the subarachnoid space (in the lateral sulci, the cysterna basalis and the longitudinal fissure of the brain) and a hemorrhagic focus $(9-\mathrm{mm})$ in the projection of the $3^{\text {rd }}$ ventricle (Figure IIA-B). MRI showed disseminated ischemic lesions in both hemispheres, in the cortical and subcortical regions and the centrum semiovales, and a saccular aneurysm of anterior communicating artery (ACA) $(7 \mathrm{~mm})$. After the patient was diagnosed with subarachnoid hemorrhage from the ACA aneurysm, embolization was performed. Within three days after the treatment, the patient reported the appearance of $\mathrm{MH}$ in the form of overlapping melodies. His results of tone audiometry were within normal limits. CT imaging repeated 6 days later showed the post-embolized state of the ACA aneurysm without bleeding in the subarachnoid space. Chronic ischemic changes were present and there were no changes in the ventricular system (Figure IIC). Results of EEG were within the normal range. A psychiatric consultation confirmed no signs of mental illness or cognitive decline. Antipsychotic treatment was administered but was later 

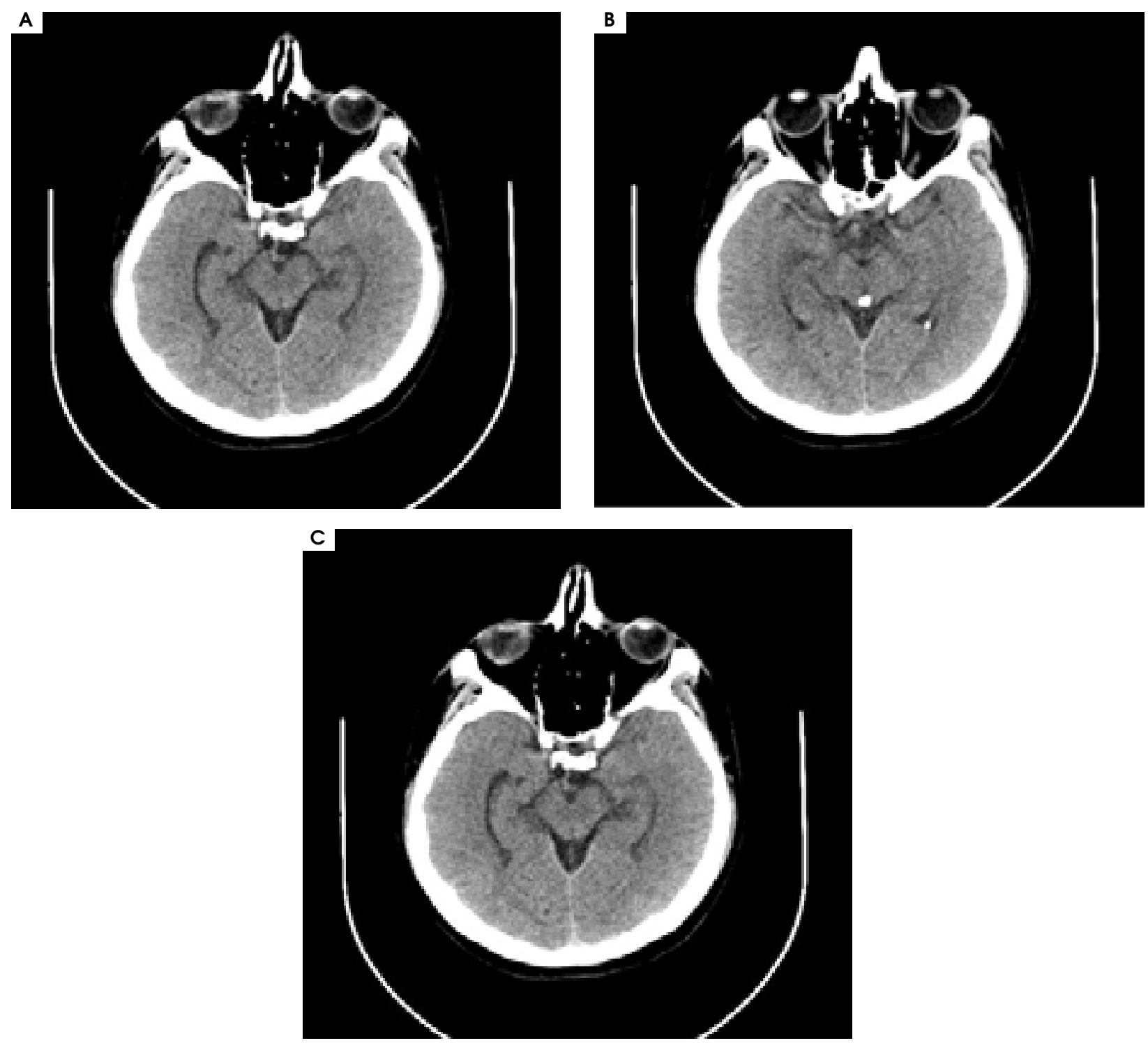

Figure II. A, B) Computed tomography (CT) imaging of a 52-year-old male showing bleeding in the lateral sulci, the cysterna basalis and longitudinal fissure of the brain, and a hemorrhagic focus (9-mm) in the projection of $3^{\text {rd }}$ ventricle. C) CT imaging 6 days after subarachnoid hemorrhage showing the post-embolized state of the anterior communicating artery aneurysm without bleeding in the subarachnoid space. Ischemic changes were present

discontinued as no improvement was observed. The progression of the auditory hallucinations to electronic and metallic sounds were reported by the patient over the next few days. Piracetam $2400 \mathrm{mg}$ /day was then administered. The auditory hallucinations subsided in approximately two weeks after the patient was discharged from the hospital. The patient no longer reported auditory symptoms at his follow-up visit three months later.

Patient 5: A 77-year-old male patient with a medical history of arterial hypertension and hyperlipidemia presented to the hospital with the primary complaint of $\mathrm{MH}$. $\mathrm{He}$ described hearing carols and lullabies interspersed with fragments of classical music, specifically melodies by Vivaldi, Beethoven, and also Charles Aznavour.
The symptoms started two years prior to his presentation to the clinic. He marked the onset to be precisely two days after the death of his wife. The patient reported gradual deterioration in hearing over the past few years. His initial referral to a psychiatrist concluded that he showed no signs of mental or cognitive disorders (MMSE $=28$ ). He was diagnosed with low mood as a result of prolonged grief and a treatment with trazodone $75 \mathrm{mg}$ /day was initiated. The subsequent neurological examination yielded no significant findings or neurological deficits. Pure tone audiometry showed moderate sensorineural hearing loss. MRI of the brain revealed disseminated ischemic lesions in both frontal, parietal and temporal lobes as well as periventricular leukoaraiosis (Figure III). EEG found no 

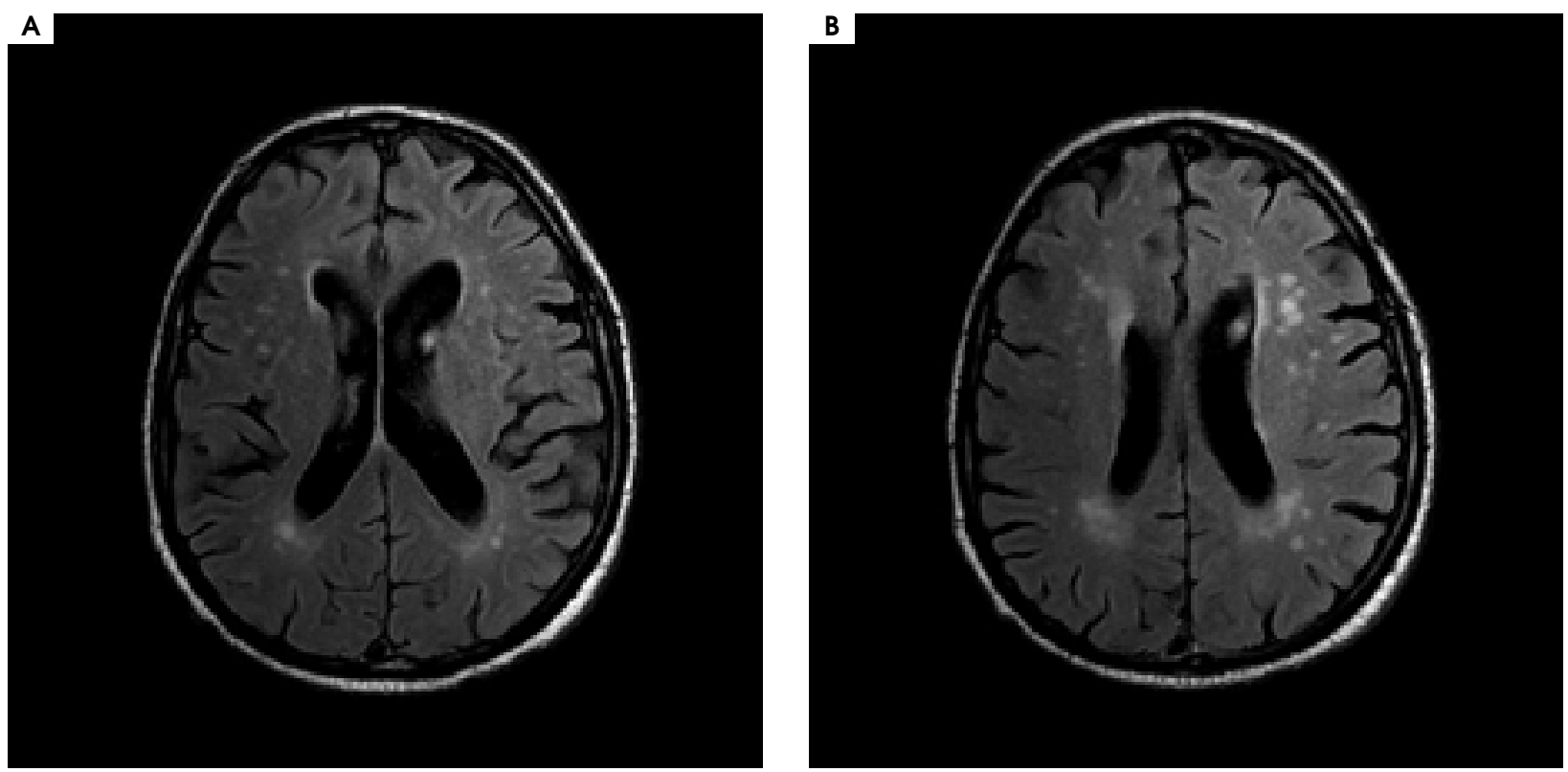

Figure III. A, B) Magnetic resonance imaging of the brain of a 77-year-old male patient showing disseminated ischemic lesions in both frontal, parietal and temporal lobes as well as periventricular leukoaraiosis

abnormalities. The treatment with carbamazepine titrated up to $800 \mathrm{mg} /$ day and vinpocetine $30 \mathrm{mg}$ /day resulted in a temporary alleviation of symptoms. When rivastigmine $3 \mathrm{mg} /$ day was added to the regimen, the symptoms resolved after a week of therapy. The patient remained in follow-up treatment and after two years he reported no recurrence of $\mathrm{MH}$.

Patient 6: A 31-year-old male patient presented to the emergency department due to right-sided hemiparesis. Neurological examination revealed diplopia, nystagmus, and right-sided extremity weakness and paresthesia. His medical history was significant for arterial hypertension and a brain stem glioma in 3 year remission after chemotherapy and radiotherapy. CT imaging of the brain showed ischemic lesions $(9 \mathrm{~mm})$ in the posterior branch of the left internal capsule and an irregular, hypodense area in the left cerebellar hemisphere $(22 \times 8 \mathrm{~mm})$. MRI of the brain showed increased signal activity (T2 and FLAIR) in the border of the thalami, the posterior lenticular nuclei, the putamen, and both cerebellar hemisphere with a small band-like enhancement. A lesion with a pathological signal in DWI was revealed on the lateral part of the medulla oblongata indicating subacute ischemia. The patient reported hearing music along with the voice of his sister originating from within his head in the week following the onset of hemiparesis. Psychological tests revealed reduced attention and ability to learn and recall new material. Treatment with risperidone $2 \mathrm{mg} /$ day was initiated but it was discontinued a month later when no improvement in symptoms was observed, and replaced with carbamazepine $400 \mathrm{mg} /$ day. After 3 months of treatment, the patient reported extended periods of silence and a progressively reduced volume of auditory hallucinations before they finally subsided in the months to follow. His mental state was assessed as normal with no other psychotic phenomena or cognitive impairment.

$\mathrm{MH}$ are a form of auditory hallucinations, a phenomenon first described by Ballinger in 1846 and subsequently identified as a rare psychopathology with less than 500 reported cases worldwide [1]. MH have heterogeneous etiologies including hearing impairment, psychosis, organic neurologic conditions including epilepsy, brain tumors, head injury, encephalitis, multiple sclerosis, and substance intoxication [4].

Auditory hallucinations after cerebrovascular events are particularly uncommon with few reported cases in recent literature. In our series of patients, auditory hallucinations often present as well-known tunes from the patient's past, including popular songs and rhythms, often consistent with previous listening experiences. All discussed patients have insight into their condition. On the one hand patients treated $\mathrm{MH}$ as intrusive and felt anxious listening to them, but on the other hand $\mathrm{MH}$ were found to be linked to pleasant memories. Only one patient's hallucinations were unfamiliar to her, suggesting that MH may comprise melodic fragments that are based on musical experience, but not necessarily reproduced entirely from musical memory.

Audiological problems represent a frequent but not necessary condition in patients presenting MH [5]. Hearing impairment was observed in four out of six cases we have reported on. This is consistent with literature reporting on a more common occurrence of $\mathrm{MH}$ among elderly 
persons with underlying hearing impairment [6]. While auditory hallucinations had been previously described as primarily happening after cerebrovascular lesions in the brainstem [3] and the temporal lobe [7], and their association with brain lateralization have been stressed [4], we found that our patients displayed disseminated ischemic changes throughout the brain - the location or the hemisphere of the lesion did not play a major role. Our patients responded to the optimization of hearing and the use of off-label anti-epileptic and cholinergic agents, consistent with those reported in current literature [5].

Auditory hallucinations can be classified as either elementary or complex. Elementary auditory hallucinations include simple sounds such as whistling and buzzing while complex hallucinations involve music, voices, or spoken words [8]. MH are uncommon in the non-psychiatric context, though have been found to exist in conditions with diverse underlying etiologies, including neurostructural lesions [4]. In a recent large case series (as reference [4]) across specialties, researchers found that neurological diagnoses associated with $\mathrm{MH}$ comprise primarily neurodegenerative disease, followed by seizure and encephalitis. Its presence in patients with cerebrovascular disease remains sparsely reported [4]. MH have been found to occur in various neuropsychiatric conditions but rarely in stroke [5].

The exact mechanism underlying $\mathrm{MH}$ is not entirely clear. It is known that temporal lobes play an important role in the perception of sounds and music as they are responsible for acoustic projections involved in the analysis of sound, tones, pitch, timbre, and rhythm which are stored as acoustic memory [9]. Kukreti described a case of a 50-year-old male with sudden onset $\mathrm{MH}$ as a result of manifestation of right temporal lobe stroke [9]. The speculative hypothesis suggests that ischemic insult to temporal lobe may lead to abnormal perception of patternsegmented sound in auditory cortex.

Although electrical stimulation of certain areas in the temporal lobes cause patients to hear music, generation of MH does not only occur in cortical areas. Acute lesions in the brainstem, thalamus, or internal capsule has also been reported to result in $\mathrm{MH}[3,4]$. The auditory symptoms, associated with brain stem stroke, include not only tinnitus or hearing loss bot also $\mathrm{MH}$ either unilateral or bilateral. These may be associated with widespread ischemia due to vertebrobasilar occlusion or large hemorrhage or with focal disease due to penetrating artery occlusion or small hemorrhage [10].

MH seem to be a phenomenon caused by heterogenous mechanisms and can not be thus regarded as a single entity [1].The human brain maintains an internal portrayal of the external environment, so sensory deprivation may result in adaptive changes within the brain. Phantom perceptions may be considered a maladaptive compensatory outcome [11]. Researchers developed the peripheral deafferentation theory, which suggests peripheral sound deprivation resulting in maladaptive amplification within cortical areas which were previously involved in musical perception [9]. A review of a large number of case studies of post-lesion hallucinations indicates that the lesion is located in the brain pathway of the sensory modality of the hallucination, which suggests compensatory over-activation of nearby brain tissue as causative in hallucinosis [10]. Scientists have also proposed a hypothesis supporting the perceptual release theory, in which a sustained level of sensory input is usually necessary to inhibit the unwanted emergence of memory traces to consciousness [12, 13]. These two theories pertain to patients with hearing impairment, which includes most but not all of the patients who present with MH. Another explanation which involves brainstem-derived $\mathrm{MH}$ suggests the release of auditory memories, which is partly under the control of pathways ascending from the reticular formation that may be disturbed in pontine lesions [14].

Evidence-based treatment guidelines for $\mathrm{MH}$ in cerebrovascular disorders have not been established due to the small number of published cases and diverse underlying pathophysiology which is not entirely clear. Various therapeutic options have been demonstrated in case reports. Not all patients need treatment, as $\mathrm{MH}$ may be self-limiting. If the patient is not in distress, even reassurance may be an adequate measure. Non-pharmacological interventions, including cognitive behavioral therapy, optimization of hearing and behavioral modifications such as listening to actual music, should be attempted first. However, no previous review has investigated the effectiveness of these interventions [5]. Off-label pharmacotherapy may include anti-epileptic, anti-depressant, anti-psychotic, or acetylcholine esterase inhibitor drugs $[1,5,10]$. While psychopharmacological and anti-epileptic medication helps treat symptoms of $\mathrm{MH}$ in patients with underlying psychiatric and epileptic disorders, limited case reports account for the use of these drugs in patients with cerebrovascular events.

$\mathrm{MH}$ remains a heterogeneous phenomenon with respect to its diverse clinical characteristics and etiologies. A thorough assessment of auditory function, neurological and psychiatric status should be carried out to classify the symptom according to their clinical perspectives. There is no single biological finding which explains the pathophysiology of MH completely. Any disruption of the musical neural network between the primary and associative cortical auditory centers can be responsible for hallucinogenesis [10]. Their diverse presentation and pathophysiology should be further explored to guide prognosis and treatment. Further clinical studies are required using the technique for mapping brain activity (e.g., functional magnetic resonance imaging fMRI or magnetoencephalography MEG) to explain pathophysiology of $\mathrm{MH}$. 


\section{Conflict of interest}

Absent.

\section{Financial support}

Absent.

\section{References}

1. Evers S, Ellger T. The clinical spectrum of musical hallucinations. J Neurol Sci 2004; 227: 55-65.

2. Stewart L, von Kriegstein K, Warren JD, Griffiths TD. Music and the brain: disorders of musical listening. Brain 2006; 129: 2533-2553.

3. Dinges M, Riemer T, Schubert T, Pruss H. Musical hallucinations after pontine ischemia: the auditory Charles Bonnet syndrome? J Neurol 2013; 260: 2678-2680.

4. Golden EC, Josephs KA. Minds on replay: musical hallucinations and their relationship to neurological disease. Brain 2015; 138: 3793-3802.

5. Coebergh JAF, Lauw RF, Bots R, Sommer IEC, Blom JD. Musical hallucinations: review of treatment effects. Front Psychol 2015; 6: 814.

6. Allanore Y, Simms R, Distler O, Trojanowska M, Pope J, Denton CP, et al. Systemic sclerosis. Nat Rev Dis Primers 2015; 1: 15002.

7. Lampl Y, Lorberboym M, Gilad R, Boaz M, Sadeh M. Auditory hallucinations in acute stroke. Behav Neurol 2005; 16: 211-216.

8. Berrios GE. Musical hallucinations. Br J Psychiatry 2018; 156: 188-194.

9. Griffiths TD. Musical hallucinosis in acquired deafness. Phenomenology and brain substrate. Brain 2000; 123: 2065-2076.

10. Braun CM, Dumont M, Duval J, Hamel-Hébert I, Godbout L. Brain modules of hallucination: an analysis of multiple patients with brain lesions. J Psychiatry Neurosci 2003; 28: 432-449.

11. Mohan A, Vanneste S. Adaptive and maladaptive neural compensatory consequences of sensory deprivation from a phantom percept perspective. Prog Neurobiol 2017; 153: 1-17.

12. Asaad G, Shapiro B. Hallucinations: theoretical and clinical overview. Am J Psychiatry 1986; 143: 1088-1097.

13. Keshavan MS, David AS, Steingard S, Lishman WA. Musical hallucinations: a review and synthesis. Neuropsychiatry Neuropsychol Behav Neurol 1992; 5: 211-223.

14. Schielke E, Reuter U, Hoffmann O, Weber JR. Musical hallucinations with dorsal pontine lesions. Neurology 2000; 55: 454-455. 